\title{
Detrended Fluctuation Analysis of Heart and Respiratory Rhythm in Atrial Fibrillation
}

\author{
Janko Zeković, Šućro Madžgalj, Mirjana M Platiša \\ Laboratory for Biosignals, Institute of Biophysics, Faculty of Medicine, University of Belgrade, \\ Belgrade, Serbia
}

\begin{abstract}
Atrial fibrillation $(A F)$ is a disorder in which heart rhythm becomes irregular and has an influence on the respiratory rhythm as well as on cardiopulmonary coupling.

We applied detrended fluctuation analysis (DFA) method to find correlation properties of heart and respiratory rhythm in order to clarify behavioral pattern of cardiopulmonary coupling in AF. Scaling exponents: $\alpha_{1}$ (short-range correlations) and $\alpha_{2}$ (long-range correlations) were calculated on $R R$ intervals and respiratory signal (Resp). Their relationship with heart/respiratory frequency was examined by regression analysis.

In comparison to the control group, there was a significant reduction of $\alpha_{1}$ and $\alpha_{2}$ of the heart rhythm in $A F$ group, while $\alpha_{2}($ Resp) was significantly increased in AF group. In regression analysis, there were significant intrasystemic relationships.

In $A F$, our results imply a compensatory mechanism in regulation of the respiratory rhythm which was not noticed in regulation of the heart rhythm. We can conclude there is a cardiopulmonary coupling in direction heart-respiratory system in $A F$, but not in the opposite direction because of impaired vagal stimulation of sinoatrial node (SA).
\end{abstract}

\section{Introduction}

Atrial fibrillation (AF) is a heart rhythm disorder in which the origin of the heart cycle is displaced from sinoatrial node (SA) onto various places in the atrium [1].

The causes of AF are not well understood, but among many proposed pathophysiological mechanisms, autonomic nervous system (ANS) regulation abnormalities are the most considered one [2,3]. It has been known for sympathetic innervation to be augmented in patients with permanent AF [4]. However, there is a lack in vagal regulation of heart rhythm leading to the absence of respiratory sinus arrhythmia-RSA [5]. RSA represents a dependence of heart rhythm by the respiratory cycle phase: accelerated pulse in the inspiration and deaccelerated in the expiration [6]. RSA originates in the brain stem having baroreceptor reflex as its underlying mechanism of action [7,8]. In addition, many data point out the phase synchronization as the interaction mechanism of cardiopulmonary system instead of RSA [9,10]. Also, recent studies have suggested that cardiopulmonary coupling is possible via reciprocal interaction mediated by ANS [11]. It should be noted that the influence of respiration process on heart rhythm is based on multiple mechanisms, while the baroreceptor reflex is the only known mechanism of the influence vice versa.

Taking into the consideration all the facts mentioned above, in this paper we quantified correlation properties of heart and respiratory rhythm using a method of detrended fluctuation analysis in order to clarify behavioral pattern of cardiopulmonary coupling in AF.

\section{Materials and methods}

Patients with permanent atrial fibrillation were included in the research (mean age 73; range 51-89 years). The control group was consisted of healthy subjects (mean age 41; range 35-45 years). Subjects were gender matched, 10 men and 3 women. Conditions of measuring were standardized [6]. Subjects were in the supination for 20 minutes of measuring ECG and the respiratory rhythm (Figure 1). ECG and the respiratory rhythm data were simultaneously recorded using 1000 $\mathrm{Hz}$ sampling frequency and 16 bit resolution by Biopac MP100 system with AcqKnowledge 3.9.1. software (BIOPAC System, Inc, Santa Barbara, CA, USA). RR(s) intervals (time intervals between heart beats) and $\mathrm{BB}(\mathrm{s})$ intervals (time intervals between breaths) were extracted from ECG and respiratory signal using OriginPro 8.6. (OriginLab Corporation, USA) software. The RR time series was formed as well as the respiratory time series (Figure 1). These time series were resampled to equidistant segments by using mean RR interval value of 
individual subject in order to apply the DFA method. Resampling was done through linear interpolation between two appropriate samples [6,12]. Analysis was conducted on overall time series of 20 minutes. In addition, RR and respiratory time series were standardized. Standardization process required extracting the mean interval value from each interval in time series and then dividing time series by its standard deviation.
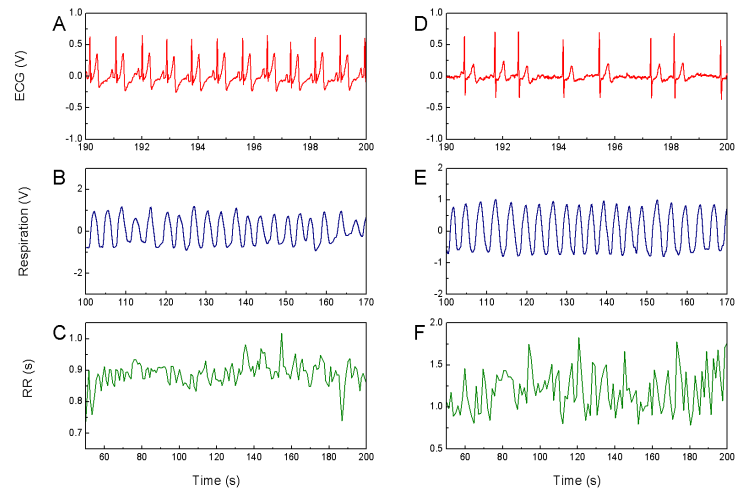

Figure 1. A - ECG of the control subject; B - respiratory time series of the control subject; $\mathrm{C}-\mathrm{RR}(\mathrm{s})$ time series of the control subject; D - ECG of the AF subject; E - respiratory time series of the AF subject; F - RR(s) time series of the AF subject.

On standardized time series the DFA method was applied [13]. Application of method includes time series integration via the following equation:

$$
y(k)=\sum_{i=1}^{k}\left[R R_{i}-R R_{\text {mean }}\right](1)
$$

Where $R_{i}$ is $i^{\text {th }} R R$ interval and $R R_{\text {mean }}$ is the mean value of $R R$ interval in given time series. Following the integration, time series was divided into equivalent segments of length $n$. In each segment of length $n$, a least square trendline was determined. A vertical coordinate of any trendline point is denoted as $y_{n(k)}$. Next step was to detrend integrated time series in every segment $n$ and calculate afterwards the square root of mean square fluctuation in segments $n$ using equation:

$$
F(n)=\sqrt{\frac{1}{N} \sum_{k=1}^{N}\left[y(k)-y_{n(k)}\right]^{2}}
$$

Logarithms of $F(n)$ and $n$ quantities were calculated and derived values were divided so that the ratio of $\log (F(n)) / \log (n)$ is characterised as a scaling exponent $\alpha$, which imlicates a linear property of the ratio ( $\alpha$ is the slope on double log graph). Fluctuation properties are denoted as scaling exponents $\alpha_{1}$ and $\alpha_{2}$.

Exponent $\alpha_{1}$ is a short-range scaling exponent, determined in the range of logarithmic values for segments of $4 \leq n \leq 16$. Exponent $\alpha_{2}$ is a long-range scaling exponent determined in the range of logarithmic values for segments of $16 \leq n \leq 250$. Values of exponent $\alpha$ indicate the following fluctuation properties: $\alpha<0.5$, anticorrelations - arranged alterations of longer and shorter intervals in the time series; $\alpha \approx 0.5$, white noise intervals are uncorrelated; $0.5<\alpha<1$, long-range correlations (power-law correlations) - longer interval is followed by another longer interval and vice versa; $\alpha=1$, $1 / f$ noise (pink noise) - value in healthy people, all regulatory mechanisms are in the harmony; $1<\alpha<1.5$, correlations exist but without a tendency to be the powerlaw correlations; $\alpha=1.5$, Brownian noise - random alteration of different intervals, integration of the white noise, small fluctuations.

The programe for calculating the mean fluctuation of integrated and detrended time series in function of the segment size $n$ is downloaded from www.physionet.org. Exponents data of RR and respiratory signal were statistically analyzed - Student $t$-test for independent (statistical significance of same exponents in a group) and dependent (statistical significance of different exponents in each group) samples. $P$ value less then 0.05 was considered as statistically significant. Results are presented as average value \pm standard error. In the end, regression analysis was made in order to determine any linear relations between the exponents and variables (heart rate - HR and breathing frequency - BF) in OriginPro 8.6. (OriginLab Corporation, USA) system. $\mathrm{HR}$ and $\mathrm{BF}$ were calculated as reciprocal mean values of $\mathrm{RR}_{\text {(mean), and }} \mathrm{BB}_{\text {(mean), respectively. Intrasystemic }}$ analyses and intersystemic analyses were made. Results are presented via graphs with Pearson correlation coefficient.

\section{Results}

In AF group, there was not statistically significant difference in HR $(p=0.39)$, but BF was significantly higher $(0.303 \pm 0.022$ vs. $0.232 \pm 0.014, p=0.01)$ in comparison to the control.

Figure 2 summarizes all comparisons of scaling exponents in the group and between them. In the AF group, $\alpha_{I}(\mathrm{RR})$ and $\alpha_{2}(\mathrm{RR})$ were statistically lower in comparison to the control $(0.496 \pm 0.027$ vs. $0.92 \pm 0.17$, $p=0.01),(0.466 \pm 0.054$ vs. $0.84 \pm 0.14, p=0.01)$, respectively. In the control group, exponent $\alpha_{1}$ (Resp) was greater in comparison to the AF group, but without statistical significance ( $p=0.33)$. But, $\alpha_{2}$ (Resp) exponent was statistically lower in the control group in comparison to the AF group $(0.14 \pm 0.06 v s .0 .271 \pm 0.075, p=0.01)$. In the control group, there was not statistically significant difference between $\alpha_{1}(\mathrm{RR})$ and $\alpha_{2}(\mathrm{RR}), \quad(p=0.421)$. However, there was statistically significant difference between $\alpha_{1}(\mathrm{Resp})$ and $\alpha_{2}$ (Resp), (0.46 \pm 0.28 vs. $0.14 \pm$ $0.06, p=0.03)$. 


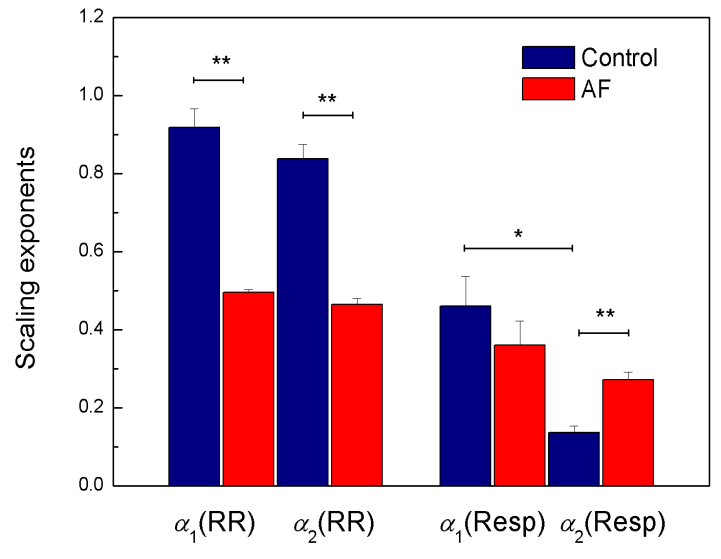

Figure 2. The average values of scaling exponents with standard errors; ${ }^{*} p<0.05$ and ${ }^{*} p p<0.01$.

In the AF group, there was not significant difference between $\alpha_{1}(\mathrm{RR})$ and $\alpha_{2}(\mathrm{RR}),(p=0.47)$, neither between $\alpha_{1}(\operatorname{Resp})$ and $\alpha_{2}(\operatorname{Resp}),(p=0.36)$.

Figure 3 shows statistically significant intrasystemic relationships. In AF group, $\alpha_{1}(\mathrm{RR})$ is related to $\mathrm{HR}$, $r=0.72, p=0.05$. In both groups, there was a relationship between $\alpha_{1}(\mathrm{Resp})$ and $\mathrm{BF}$ (AF, $p=0.04$ and the control group, $r=-0.62, p=0.02$ ).
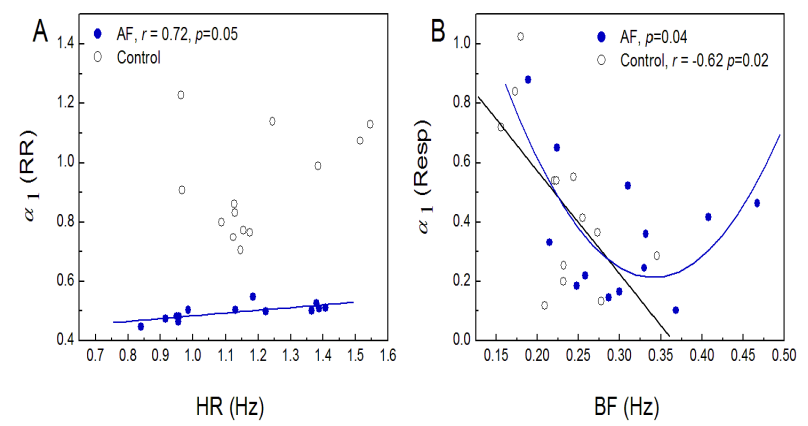

Figure 3. Intrasystemic regression analysis: A. $\alpha_{1}(\mathrm{RR}) v s$. HR, $y=0.093 x+0.39, r=0.72, p=0.05$; B. $\alpha_{1}$ (Resp) $v s . \mathrm{BF}, \mathrm{AF}$ group, $y=20.3 x^{2}-13.8 x+2.6, \mathrm{R}=0.68, p=0.04$ and the control group, $y=-3.47 x+1.27, r=-0.62, p=0.02$.

In intersystemic regression analysis, there was not any significant relationships except for noted tendency of $\alpha_{2}$ (Resp) to be related to HR in AF, $r=0.50, p=0.08$.

\section{Discussion}

There were changes in regulatory mechanisms of heart and respiratory rhythm in atrial fibrillation and in cardiopulmonary coupling as well.

In the control group there was not statistically significant difference between $\alpha_{1}(\mathrm{RR})$ and $\alpha_{2}(\mathrm{RR})$, and the values of exponents were close to 1 i.e. $1 / f$ noise, which is the property of healthy cardiac rhythm $[13,14]$. However, the results showed that there was not a crossover between $\alpha_{1}(\mathrm{RR})$ and $\alpha_{2}(\mathrm{RR})$ exponents in $\mathrm{AF}$ group either, while decreased values indicated that the both types of correlations were diminished (the regulatory mechanism was equally dimished in both types). Gained result for dimished $\alpha_{1}(\mathrm{RR})$, as a pathological feature, was in the accordance with previous conducted studies [13].

In addition, regression analysis of intrasystemic relationships, $\alpha_{1}(\mathrm{RR})$ vs. HR showed statistically significant relationship in the AF group, but not in the control group. This might be explained by different pattern of HR regulation because in AF parasympathetic regulation lacks i.e. sympathetic activity has an influence on $\alpha_{1}(\mathrm{RR})$ value [6].

Although lower values of $\alpha_{1}(\mathrm{RR})$ are in the accordance with previous studies [13], lesser values of $\alpha_{2}(\mathrm{RR})$ are not. Having in mind there is no proof of respiratory sinus arrythmia in AF [6], lesser value of $\alpha_{2}$ (RR) might be explained by the absence of vagal stimulation and any other neural compensatory mechanism considering low levels of $\alpha_{1}(\mathrm{RR})$.

Respiratory frequency was statistically greater in $\mathrm{AF}$ group in comparison to the control group, which might be explained by compensatory mechanisms of baroreceptor reflex which is dominant with Bainbridge reflex in cardiopulmonary coupling $[7,8]$.

Comparing values of $\alpha_{1}$ (Resp) exponent between the groups, there was not noticed statistically significant difference. Tendency of $\alpha_{1}$ (Resp) to be lower in the AF group could be explained by indirect influence of the heart pathology having more dominant influence than any other regulatory mechanism of the respiratory rhythm. However, there was statistically significant difference in long-range correlation between the groups with higher $\alpha_{2}(\operatorname{Resp})$ value in AF group, which could be explained by higher respiratory frequency in this group. More precisely, $\alpha_{2}$ (Resp) was in the range of anticorrelations in the control group (arranged pattern of fluctuation of shorter and longer intervals between each breath). But, in the AF group $\alpha_{2}$ (Resp) was in the range of white noise (uncorrelated intervals) and this alteration of shorter and longer intervals in respiratory signal could not be detected because of higher respiratory frequency values in this group.

Comparing $\alpha_{1}$ (Resp) and $\alpha_{2}$ (Resp) in the control group, there was noticed statistically significant difference with lower value of $\alpha_{2}$ (Resp) exponent (anticorrelations) and this pattern of long-range correlation of the respiratory rhythm reflects a harmony of multiple regulatory mechanisms of the respiratory rhythm [11]. In the AF group, there was not noticed statistically significant difference between $\alpha_{1}$ (Resp) and 
$\alpha_{2}$ (Resp), which can be once more explained by more dominant influence of the heart on the respiratory system, i.e. in order to maintain appropriate ventilation-perfusion ratio.

Moreover, regression analysis of intrasystemic relationships, $\alpha_{1}$ (Resp) vs. BF showed a decreasing trend in short-range correlation with the increase of respiratory frequency. Intrasystemic analysis of $\alpha_{2}$ (Resp) vs. BF did not show statistically significant relationship. Taking this into the consideration, we conclude there is a relationship between $\alpha_{1}$ (Resp) and the BF so that when BF increases, $\alpha_{1}$ (Resp) decreases in both groups, but in AF group only to some value of $\mathrm{BF}$, when its further increase leads to the increase of $\alpha_{1}$ (Resp) as well. This can be explained by altered regulation of the heart rhythm. Furthermore, $\alpha_{2}$ (Resp) was independent of the BF in both groups - also possible explanation lies in multiple regulatory mechanisms of the respiratory rhythm [11]. This was in accordance with anticorrelations of $\alpha_{2}$ (Resp) exponent in the control, even though this exponent was slightly increased in AF group (this increase is a consequence of altered heart rhythm which can be seen from intersystemic regression analysis $\alpha_{2}$ (Resp) vs. HR).

However, there was not statistically significant relationship of $\alpha_{1}$ (Resp) vs. HR in regression analysis. This might be due to the fact that slightly increased values of $\mathrm{HR}$ in the control was one of the mechanisms which in collaboration with others regulated the respiratory rhythm (with the assumption this is the case when there is no pathological conditions in the organism). Also, a longer time interval was needed for higher values of HR to become more dominant over other regulatory mechanisms of the respiratory rhythm in sick people. Latter implicates a possible explanation for noticed tendency of $\alpha_{2}$ (Resp) to be related to HR.

\section{Conclusion}

Considering the obtained results, there is an implication of a compensatory mechanism in regulation of the respiratory rhythm in atrial fibrillation. Still, we did not notice the existence of another neural compensatory mechanism in regulation of the heart rhythm. We can conclude there is a cardiopulmonary coupling in direction heart-respiratory system in AF, but not in the opposite direction because of impaired neural cardiac control i.e. vagal stimulation of sinoatrial node.

\section{Acknowledgements}

The authors would like to thank prof. S. Pavlović and dr N. Radovanović for their help in obtaining the AF data.

\section{References}

[1] Marchlinski F. The Tachyarrhythmias. In: Joseph Loscalzo, editor. Harrison's Cardiovascular Medicine. New York: McGraw Hill Medical; 2010. p.147-178

[2] Lau HD, Schotten U, Mahajan R et al. Novel mechanisms in the pathogenesis of atrial fibrillation: practical applications. Eur Heart J 2016; 37: 1573-1581.

[3] Opacic D, van Bragt KA, Nasrallah HM et al. Atrial metabolism and tissue perfusion as determinants of electrical and structural remodelling in atrial fibrillation. Cardiovasc Res. 2016; 109: 527-541.

[4] Platiša MM, Bojić T, Pavlović SU et al. Generalized Poincaré Plots-A New Method for Evaluation of Regimes in Cardiac Neural Control in Atrial Fibrillation and Healthy Subjects. Front Neurosci. 2016; 10:38.

[5] Pitzalis MV, Massari F, Forleo C et al. Respiratory systolic pressure variability during atrial fibrillation and sinus rhythm. Hypertension 1999; 34: 1060-1065.

[6] Platiša MM, Bojić T, Pavlović SU et al. Uncoupling of cardiac and respiratory rhythm in atrial fibrillation. Biomed Tech (Berl). 2016; 61: 657-663.

[7] Farmer DG, Dutschmann M, Paton JF et al. Brainstem sources of cardiac vagal tone and respiratory sinus arrhythmia. J Physiol. 2016; 594: 7249-7265.

[8] Karemaker JM. Counterpoint: respiratory sinus arrhythmia is due to the baroreflex mechanism. J Appl Physiol (1985). 2009; 106: 1742-3

[9] Schäfer C, Rosenblum GM, Kurths J, Abel HH. Heartbeat synchronized with ventilation. Nature 1998; 392: 239-240

[10] Lotrič MB, Stefanovska A. Synchronization and modulation in the human cardiorespiratory system. Physica A 2000; 283: 451-461.

[11] Dick TE, Hsieh YH, Dhingra RR et al. Cardiorespiratory coupling: common rhythms in cardiac, sympathetic, and respiratory activities. Prog Brain Res. 2014; 209: 191-205.

[12] Kapidžić A, et al., Nonlinear properties of cardiac rhythm and respiratory signal under paced breathing in young and middle-aged healthy subjects. Med Eng Phys. 2014 ;36(12):1577-84.

[13] Peng CK, Havlin S, Stanley HE, Goldberger AL. Quantification of scaling exponents and crossover phenomena in nonstationary heartbeat time series. Chaos. 1995; 5: 82-7.

[14] Goldberger AL, Amaral LA, Hausdorff JM et al. Fractal dynamics in physiology: Alterations with disease and aging. PNAS 2002; 99: 2466-2472.

Address for correspondence.

Janko Zeković, undergraduate student zekovic.janko@gmail.com; mirjana.platisa@gmail.com Laboratory for Biosignals, Institute of Biophysics Faculty of Medicine, University of Belgrade Višegradska 26-2, pp22, 11102 Belgrade, Serbia 